\title{
Detection of Pathological Myopia by PAMELA with Texture-Based Features through an SVM Approach
}

\author{
Jiang Liu ${ }^{1}$, Damon W.K. Wong ${ }^{1}$, Joo Hwee Lim ${ }^{1}$, Ngan Meng Tan ${ }^{1}$, \\ Zhuo Zhang1, Huiqi Li ${ }^{1}$, Fengshou Yin ${ }^{1}$, Benghai Lee', Seang Mei Saw ${ }^{2}$, \\ Louis Tong ${ }^{3}$, Tien Yin Wong ${ }^{3}$ \\ ${ }^{1}$ Institute for Infocomm Research, $A^{*}$ STAR, Singapore \\ ${ }^{2}$ National University of Singapore \\ ${ }^{3}$ Singapore Eye Research Institute
}

\begin{abstract}
Pathological myopia is the seventh leading cause of blindness worldwide. Current methods for the detection of pathological myopia are manual and subjective. We have developed a system known as PAMELA (Pathological Myopia Detection Through Peripapillary Atrophy) to automatically assess a retinal fundus image for pathological myopia. This paper focuses on the texture analysis component of PAMELA which uses texture features, clinical image context and support vector machine-based classification to detect the presence of pathological myopia in a retinal fundus image. Results on a test image set from the Singapore Eye Research Institute show an accuracy of $87.5 \%$ and a sensitivity and specificity of 0.85 and 0.90 respectively. The results show good promise for PAMELA to be developed as an automatic tool for pathological myopia detection.
\end{abstract}

Keywords: pathological myopia, peripapillary atrophy, computer aided detection

\section{INTRODUCTION}

Myopia is a visual condition of the eye, defined as a refractive error of more than 0.5 dioptres (D), in which the images are not focused on the retina but rather in front of it, causing visual blurring. Studies in the United States have shown that the prevalence of myopia is approximately $25 \%$ [1]. In Singapore, the prevalence of myopia is even higher, at more than $70 \%$ [2]. The social and economic impact of myopia is a considerable issue in public health today.

A more serious myopic condition, known as pathological or degenerative, myopia, is different from simple refractive myopia. Pathological myopia is defined as an extremely high level of shortsightedness caused by pathologic axial elongation [3] of the eyeball. In the United States, the disease affects $2 \%$ of the population, and is the seventh leading cause of blindness. [4,5]. Further, it has been reported that there is increased prevalence of the disease particularly in Asian populations [2]. 
The increasing prevalence of myopia, particularly among children [6] is a cause for concern, since severity of myopia is potentially proportional to the risk of pathological myopia [7]. It is thus important to develop screening and management systems to detect the disease early for potential interventional measures to be taken [8]. However, current methods of detection remain highly reliant on visual acuity testing, which are manual, time-consuming and subject to the individual patient's evaluation of visual acuity and may be inconsistent in young patients. There is hence a great need to objectively and automatically detect the presence and track the progress of pathologic myopia.

Besides a worsening of vision, pathological myopia is typically accompanied by visual features which can be observed on a retinal fundus image. Such cues include straightened and stretched vessels, temporal peripapillary atrophic crescent, tilting of the optic disc, posterior staphyloma, lacquer cracks in the Bruch's membrane, geographic areas of atrophy of the retinal pigment epithelium and choroids, subretinal haemorrhage, and choroidal neovascularisation [9].

In this work, we report the development of PAMELA (Pathological Myopia Detection Through Peripapillary Atrophy), a system for the automatic detection of pathological myopia. Peripapillary atrophy (PPA) is a pathological cue highly associated with pathological myopia. In particular, the focus of the present work is on the texture-based component of PAMELA. The component automatically takes in a retinal fundus image, and performs region of interest (ROI) extraction and detection of the optic nerve head. Subsequently, texture-based metrics are generated, categorized and grouped into zones for context-based generation of features. These features will be used in a support vector machine to determine the presence of PPA, and correspondingly, pathologic myopia.

Section I has briefly introduced described pathological myopia and the motivations for its automated detection. Section II provides a description of the overall framework of the system, and how the initial retinal fundus image is processed for region of interest extraction, optic nerve head determination, texture feature generation, and SVM-based classification. Section III describes experiments carried out on an image set of images and the performance of the system, and Section IV provides an overall conclusion to the paper.

\section{METHODS}

In a person suffering from pathological myopia, one of the key visual features is the presence of peripapillary atrophy, which can be described as a "splotchy", rough and irregular region on the periphery of the optic disc, as shown in Figure 1. The distinct appearance of PPA motivated the design of a system which relies on texture analysis. In the system shown in Figure 2, which is a key component of the overall PAMELA framework, texture-based features are used to determine the presence or absence of PPA in a retinal image. A key feature of the framework is the incorporation of clinical knowledge-based heuristics throughout the system, which helps to improve the accuracy and efficiency of the PPA detection. The following subsections describe each of the various modules in the system shown in Figure 2. 


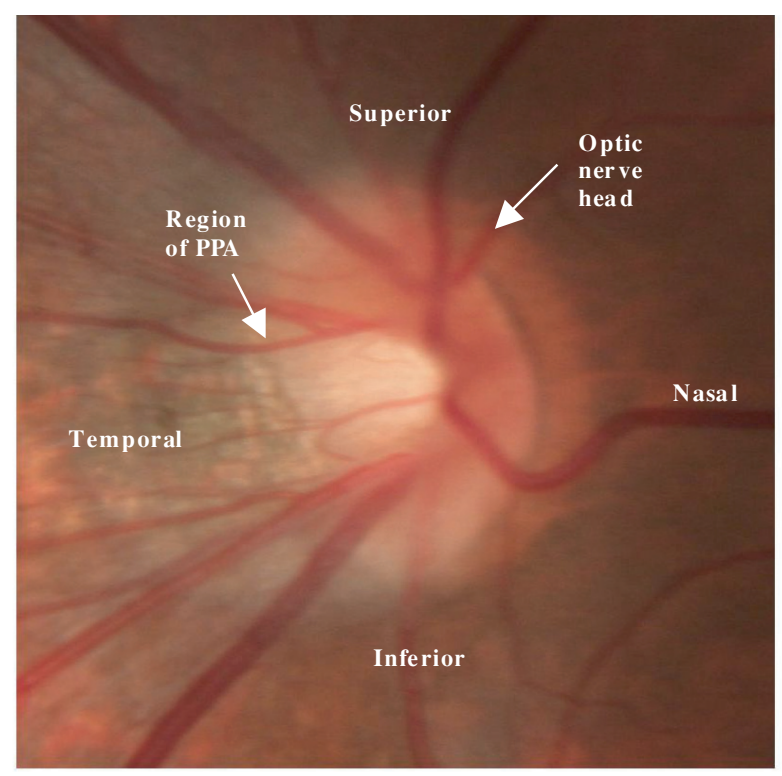

Figure 1. A retinal fundus image of a right eye. Annotations indicate key features of the image. The nasal and temporal sectors are flipped for a left eye.

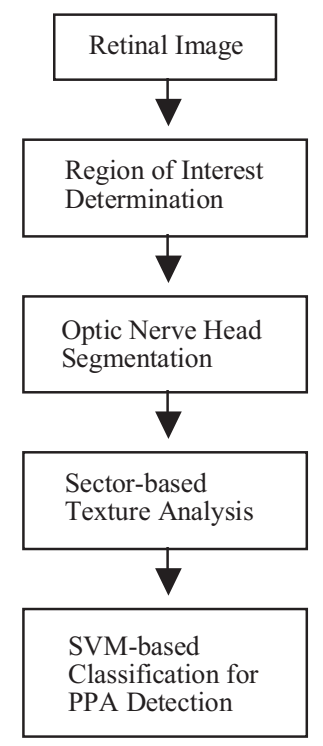

Figure 2. System for the detection of PPA using texture features and SVM classification. This is a key component of the overall PAMELA framework. 


\section{A. ROI Detection}

In a typical retinal fundus image with a $45^{\circ}$ field, as seen in Figure 3(a), the optic nerve head typically takes up less than $20 \%$ of the entire image. By limiting the region of analysis in the subsequent steps, the computational cost can be reduced, resulting in a more efficient computation. Furthermore, by defining the ROI to only focus on the optic nerve head, errors resulting from other image artifacts, retinal physiology or other pathologies can be minimized.

In our system, a histogram-based approach is employed to exploit the color characteristics of the optic nerve head in the red channel, as can be seen in Figure 3(b). In particular, since the optic nerve head has the highest intensity in the red channel, a histogram is first constructed using the red channel, and pixels contributing to the highest $1 \%$ are first selected. The object with the largest number of pixels is then denoted as the approximate optic nerve head. A possible cause of error in this approach is the occasional presence of a characteristic bright crescent located on the boundary of the retinal image, due to misalignment in the retinal camera between the eye and the lens system. To avoid this error, a filter is used to eliminate objects in which the height is more than three times the width, since the optic nerve head is approximately circular or slightly vertical in nature.

Once the approximate optic nerve head location has been detected, the centroid of the object is determined and an $800 \times 800$ pixel ROI around this centroid is utilized for subsequent analysis.

\section{B. Optic Nerve Head Detection}

Using the ROI from the previous step, the optic nerve head is segmented using a levelset algorithm based on [9]. Known as the variational formulation of the level-set algorithm, this algorithm avoids the need of costly computation for re-initialization during the evolution process. This is done by modifying the energy function $\varepsilon(\phi)$ to include an internal energy term $\mu P(\phi), \mu>0$ which penalizes the deviation of the evolving function $\phi$ from a signed distance function, and $\varepsilon_{m}(\phi)$, an external energy term that drives the zero level set towards the object boundaries. The resulting energy term is thus given by

$$
\varepsilon(\phi)=\mu P(\phi)+\varepsilon_{m}(\phi)
$$

and the level set function consequently is able to avoid shocks resulting in sharp or flat boundaries. Further details of this level-set formulation can be found in [10].

Before applying the above method, the ROI is first blurred via a 2D gaussian filter so as to reduce the visibility of structures within the optic disc and to avoid erroneous segmentations. The variational level-set approach is then used to obtain the disc. It was observed that the presence of blood vessels traversing the disc boundary was a potential cause of error in the disc boundary, contributing to the rough edges of the detected boundary. To avoid this, direct ellipse fitting [11] was applied onto the level-set contour, which helped to mitigate the uneven edges of the optic nerve head. The same approach described here has been taken in our previous work of ARGALI for glaucoma detection $[12,13]$. 
Generally, this approach is able to effectively segment the optic nerve head and provide a good approximation of the optic disc boundary. In certain conditions, such as in severe optic disc tilt, the optic disc can potentially be under-segmented, particularly in the nasal sector. However, the impact on the results is minimal as the difference between the actual optic disc and the under-segmented area is small. Furthermore, the enlarged area of the optic disc in practice adds to the buffer zone described below. For images where the accurate detection of the optic nerve head is critical, the optic disc location may also be manually adjusted.

\section{Sector-based Texture Analysis}

(1) Entropy Transform

Whereas the red channel was used in the previous steps, in the texture analysis for PPA, the blue channel is used owing to its greater visibility. To further increase the delectability of PPA, the histogram of the ROI is automatically adjusted such that only $1 \%$ of the data is saturated at the bottom $1 \%$ and top $1 \%$ of all the pixel values. This helps to raise the contrast and corresponding visibility of the PPA, since PPA has a granulated, uneven visual appearance.

Entropy analysis on the ROI is then performed. Entropy is a measure of the roughness in an image [13], defined as

$$
\varepsilon=\sum p \log _{2} p
$$

where $p$ is the normalized intensity histogram for the $9 \times 9$ pixel kernel applied throughout the ROI, generating an entropy value for each pixel in the ROI. Higher values of $\varepsilon$ indicate a rougher, coarse texture indicative of PPA, while lower values of $\varepsilon$ imply a less uneven, smoother appearance uncharacteristic of PPA. The entropy transform thus results in a texture map indicative of roughness in the ROI. The texture map is further categorized by rounding off and grouping the entropy values from 0 to 5, as shown in Table 1. An example of the texture map generated from the described entropy analysis is shown in Figure 4(a).

Table 1. Classification of values

\begin{tabular}{cc}
\hline Entropy values & Classification \\
\hline$\varepsilon<1$ & 1 \\
$1 \leq \varepsilon<2$ & 2 \\
$2 \leq \varepsilon<3$ & 3 \\
$3 \leq \varepsilon<4$ & 4 \\
$4 \leq \varepsilon<5$ & 5
\end{tabular}




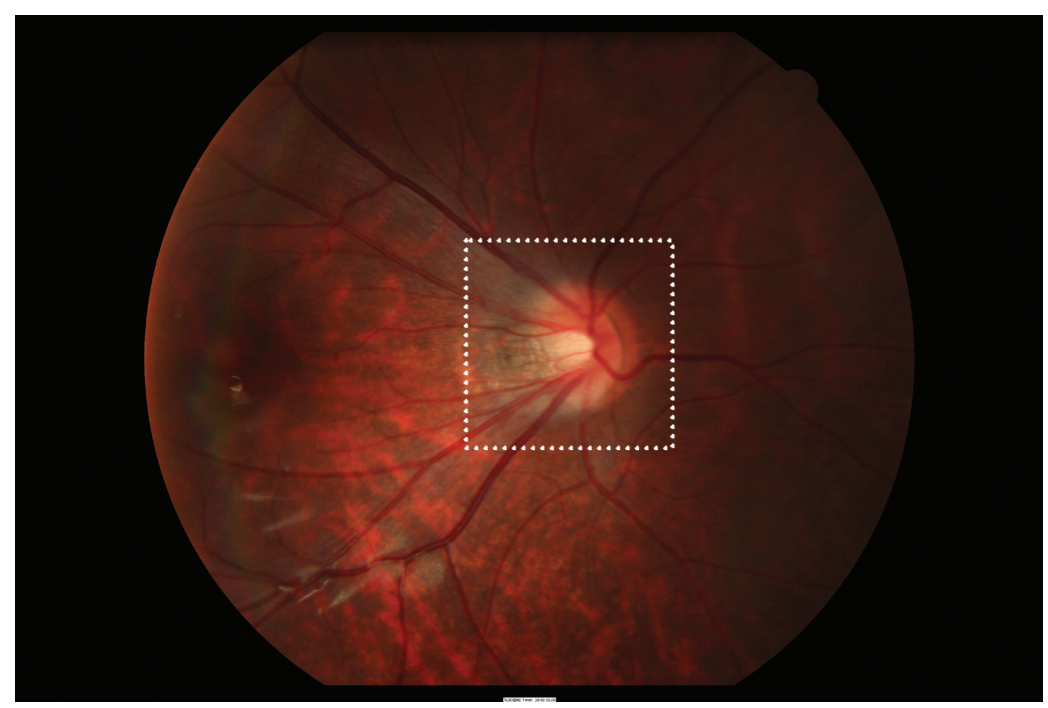

(a)

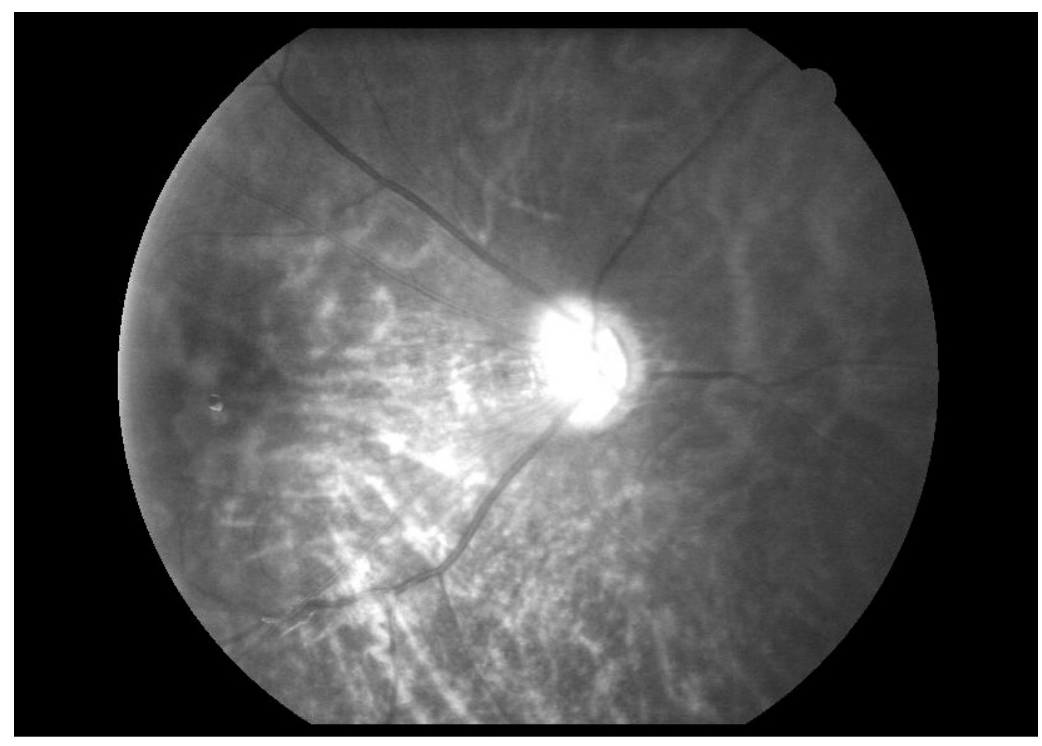

(b)

Figure 3. (a) Color and (b) red channel images of a retinal fundus image. The box indicates the ROI extracted from these images, shown in Figure 1. 


\section{(2) Sectorization of the ROI}

A retinal image can be subdivided into different sectors, or zones, based on the location of the zone with respect to the centre of the optic nerve head, and is typically described with the following terms: inferior (the lower region), superior (the upper region), nasal (the region nearer the nose) and temporal (the region nearer the temple), as indicated in Figure 4(b) for a left eye. The horizontal zones are flipped for a right eye. In pathological myopia, PPA tends to occur in the temporal region of the optic nerve head periphery, and such contextual information can be helpful in the detection of PPA by subdividing the extra-peripheral area of the optic nerve head into such zones, and considering the texture-based features for each zone. Only the region outside the optic nerve head is considered, since PPA tends to occur outside the optic nerve head. An additional buffer zone is also defined around the disc boundary, due to the high contrast of the physiological disc boundary. The high contrast is a potential source of error which can mislead the measures of roughness. By taking into account only the regions beyond the buffer zone, a more accurate reading of the PPA can be achieved. This would also help to reduce the effect of false detection due to other physiological structures such as the boundary of the optic nerve head or vasculature. An example of how the zones are defined together with the corresponding buffer zones is shown in Figure 4(b).

\section{(3) Feature Generation}

Using the zones defined above in 2), and illustrated in Figure 4(b) below, the results from the entropy transform are then utilized to generate the following features:

$$
\left.\begin{array}{l}
s_{z} \\
\mu_{z}=\frac{s_{z}}{N_{Z}} \\
n_{4 Z} \\
d_{4 z}=\frac{n_{4 Z}}{N_{Z}} \\
n_{5 Z} \\
d_{5 z}=\frac{n_{5 Z}}{N_{Z}}
\end{array}\right\} \quad Z=\{I, S, N, T\}
$$

where, for each zone $Z=\{$ Inferior, Superior, Nasal, Temporal $\}, s_{Z}$ is the entropy score, $N_{Z}$ is the number of pixels, $\mu_{Z}$ is the average entropy score, $n_{4 Z}$ is the number of pixels with texture class of 4 or more (based on Table I), $d_{4 Z}$ is the density of class 4 or higher pixels, $n_{5 Z}$ is the number of pixels with texture class of 5 or more (based on Table I), and $d_{5 Z}$ is the density of class 5 or higher pixels. In addition, since the PPA has a high tendency to occur on the temporal side and a low tendency of occurrence on the nasal side, we also include relativistic metrics: 


$$
\begin{aligned}
& s_{T N}=s_{T} / s_{N} \\
& \mu_{T N}=\mu_{T} / \mu_{N} \\
& n_{4 T N}=n_{4 T} / n_{4 N} \\
& d_{4 T N}=d_{4 T} / d_{4 N} \\
& n_{5 T N}=n_{5 T} / n_{5 N} \\
& d_{5 T N}=d_{5 T} / d_{5 N}
\end{aligned}
$$

where the ratio of the individual features for the temporal and nasal zones are calculated. The combination of the individual and relativistic metrics generates a total of 30 features for each retinal fundus image.

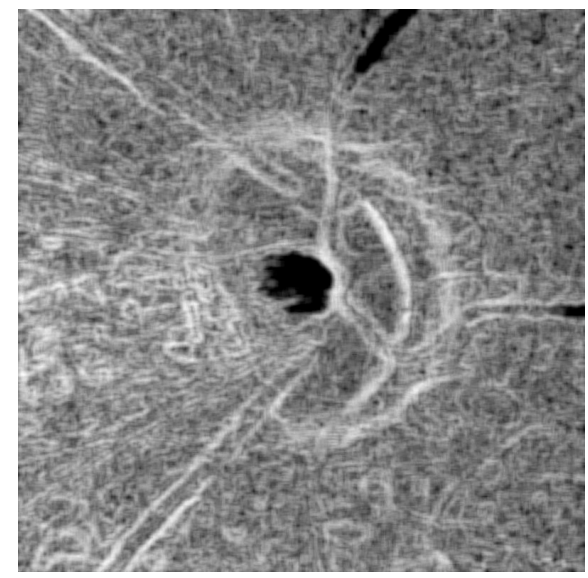

(a)

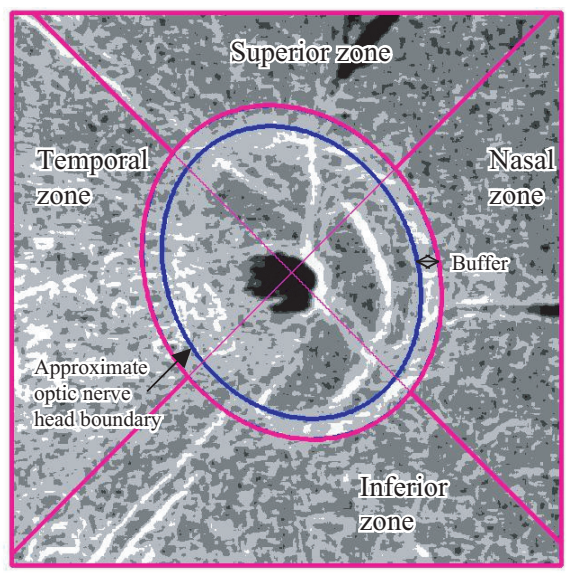

(b)

Figure 4. (a) Entropy transform and (b) categorized entropy values of the results shown in (a), using Table I, (b) also shows the different zones and the buffer layer around the detected optic nerve head.

\section{SVM Classification}

Subsequently, these features are employed as inputs in a Support Vector Machine (SVM) classifier. SVMs are a type of machine learning method in which is the aim is to learn the relationships (model) between the attributes (features) of a sample and its label (class). Developed by Vapnik et al. [15] initially for character recognition problems, SVMs have been used in many classification and regression applications including medical image analysis tasks. In SVM, the focus is on determining the maximal separating hyperplane between classes. Given a set of $l$ training instances $\left(\mathbf{x}_{\mathbf{i}}, y_{i}\right), i=1, \ldots, l$, where the $\mathbf{x}_{\mathbf{i}}$ is the feature vector and $y_{i}$ is the target label 
$y \in\{1,-1\}$, the implementation of SVM by Vapnik [14] involves the solution of the quadratic programming problem in the standard formulation:

$$
\begin{array}{ll}
\min _{\mathrm{w}, \mathrm{b}, \boldsymbol{\xi}} & \frac{1}{2} \mathrm{w}^{T} \mathrm{w}+\mathrm{C} \sum_{\mathrm{i}=1}^{1} \xi_{i} \\
\text { subject to } & y_{i}\left(\mathrm{w}^{T} \phi\left(x_{i}\right)+b\right) \geq 1-\xi_{i} \\
& \xi_{i} \geq 0
\end{array}
$$

where $\mathrm{w}$ and $b$ are the coefficients of this maximal hyperplane, $\xi$ is the slack variable associated with tolerating errors and $C$ is a user-defined penalizing cost of including such errors. The instances are often not linearly separable, and this requires transformation of the feature space using a basis function, such as a radial basis function. Once the model has been trained, classification decisions are performed using $\operatorname{sgn}\left(w^{T} x+b\right)$.

In the PPA assessment system, the 30 feature vectors obtained in the feature generation step is used as an input for the SVM classifier, and the output of the system produces a classification results for the presence or absence of PPA.

\section{EXPERIMENTS AND RESULTS}

In order to evaluate the performance of the system, an image set of 80 retinal fundus images from the Singapore Cohort Study of the Risk Factors for Myopia (SCORM) were obtained from the Singapore Eye Research Institute (SERI), which was divided into a group of 40 images with pathological myopia, and another group of 40 clinically normal images. Each retinal fundus image has a resolution of $3504 \times 2336$ pixels. An ophthalmologist assessed each image, whereupon the presence of PPA was confirmed in the patients with pathological myopia.

To train the SVM model, 20 images from the PPA set and 20 images from the normal set were randomly selected to form the training set. The remaining images from the sample set were used to form the test set. For the SVM implementation, we made use of C-SVM algorithm from the LIBSVM toolbox [16], selecting the radial basis function as the kernel function. For system validation, we conducted leave-one-out crossvalidation using the training set. Two rounds of optimization were carried out to determine the optimum performing SVM parameters, the first being a coarse search and the subsequent step being a fine search based on the prior results. Using the optimal parameters for the SVM model, we achieved a cross-validation accuracy of $82.5 \%$ for the detection of PPA on the training set only.

Subsequently, the trained SVM model was used to evaluate PPA detection on the test set of 40 images, achieving a detection accuracy of $87.5 \%$. To further assess the performance, we determined the specificity and sensitivity of the system under test. Sensitivity, defined as the number of true positives divided by the sum of true positives and false negatives, indicates how well a test is able to identify actual positives. Specificity, defined as the number of true negatives divided by the sum of true negatives and false positives, indicates how well a test is able to correctly identify negatives. 
Based on the training set, the system was able to achieve a sensitivity and sensitivity of 0.85 and 0.90 , respectively.

\section{CONCLUSION AND FUTURE WORK}

Pathological myopia is a serious condition of the eye and is the seventh leading cause of blindness worldwide. It is important to be able to detect the disease early for potential interventional measures to be undertaken. However, current methods are subjective and manual. We have presented an automated system for the detection of pathological myopia. The system, PAMELA, developed with medical doctors and incorporating clinical contextual knowledge, takes in a retinal fundus image and analyzes it for the presence of peripapillary atrophy, a visual feature highly correlated with the disease. Key features of the system involve segmentation of the optic nerve head, generation of texture features, grouping of these features into clinically-relevant zones, and a SVM classifier trained for classification on the detection of PPA. The results show a detection accuracy of $87.5 \%$, with a sensitivity of 0.85 and specificity of 0.90 .

Future developments of the system include improving the accuracy of the optic disc detection, and differentiation between different types of PPA. Also, in the current system, the detection of PPA is a binary result, indicating only absence or presence of PPA. By providing a grade or scale, the extent and progression of PPA can be better tracked, and the risk of pathological myopia can be better determined.

Furthermore, as pathological myopia is defined by a number of other pathological visual features, we are working towards expanding the scope of PAMELA to detect and include these features in the SVM framework. This would help to enhance the accuracy of PAMELA as a viable tool for automatic pathological myopia detection, which could be used in screening to detect previously undiagnosed cases in as early a stage as possible.

\section{REFERENCES}

[1] R.D. Sperduto, D. Seigel, J. Roberts, et al, "Prevalence of myopia in the United States," Arch Ophthalmol, Vol. 101, pp. 405-407, 1983.

[2] T.Y. Wong, P. Foster, J. Hee, et al, "Prevalence and risk factors for refractive errors in adult Chinese in Singapore," Invest Ophthalmol Vis Sci, Vol. 41, pp.2486-2494, 2000.

[3] T. Tokoro, "On the definition of pathologic myopia in group studies," Acta Ophthalmol. Suppl., 185, pp. 107-108, 1988.

[4] M. Secretan., D. Kuhn, G. Soubrane, G. Coscas, "Long-term visual outcome of choroidal neovascularization in pathologic myopia: natural history and laser treatment," Eur J Ophthalmol, Vol. 7, pp. 307-16, 1997.

[5] H.E. Grossniklaus, and W.R. Green, "Pathologic findings in pathological myopia," Retina, Vol. 12, pp. 127-33, 1992.

[6] S.M. Saw, L. Tong, W.H. Chua, et al, "Incidence and Progression of Myopia in Singaporean School Children," Invest Ophthalmol Vis Sci., Vol. 46, pp.51-57, 2005.

[7] S.M. Saw, "How blinding is pathological myopia?" Br. J. Ophthalmol., Vo.1 90, pp. 525-526, 2006.

[8] W.M. Chan, M. Ohji, T.Y.Y. Lai, D.T.L. Liu, Y. Tano, D..S.C. Lam, "Choroidal neovascularisation in pathological myopia: an update in management”, Br. J. Opth., Vol. 89(11), pp 1522-1528.

[9] Y.F. Shih, T.C. Ho, C.K. Hsiao, L.L.-K. Lin, "Visual outcomes for high myopic patients with or without myopic maculopathy: a 10 year follow up study,” Br. J. Opth, Vol. 90, pp 546-550. 2006. 
[10] C. Li, C. Xu, C. Gui, M. D. Fox, "Level set evolution without re-initialization: a new variational formulation", Proc. of the 2005 IEEE Computer Society Conference on Computer Vision and Pattern Recognition, 2005.

[11] A. Fitzgibbon, M. Pilu, R. B. Fisher, "Direct least squares fitting of ellipses", IEEE Transactions on Pattern Analysis and Machine Intelligence, Vol. 21, pp. 476 - 480, 1999.

[12] I.J. Liu, D.W.K. Wong, J.H. Lim, H. Li, N.M. Tan, Z. Zhang, T. Y Wong, R. Lavanya, “ARGALI : An Automatic Cup-To-Disc Ratio Measurement System For Glaucoma Analysis Using Level-Set Image Processing”, 13th International Conference on Biomedical Engineering (ICBME2008), 2008.

[13] J. Liu., D.W.K. Wong, J. H. Lim., H. Li, F.Yin, X. Jia, K. L. Chan, N. M. Tan. T. Y. Wong., “ARGALIan Automatic cup-to-disc Ratio measurement system for Glaucoma detection and AnaLysIs framework", Proceedings of SPIE, Vol. 7260, 72603K (2009)

[14] R.C. Gonzalez, R.E. Woods, S.L. Eddins, Digital Image Processing Using MATLAB, New Jersey, Prentice Hall, 2003.

[15] V. Vapnik, The Nature of Statistical Learning Theory, Second Edition, Springer, New York, 2001.

[16] C.-C. Chang and C.-J. Lin, LIBSVM: a library for support vector machines, 2001. 



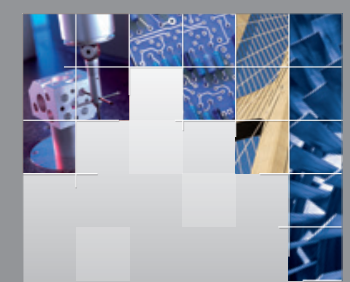

\section{Enfincering}
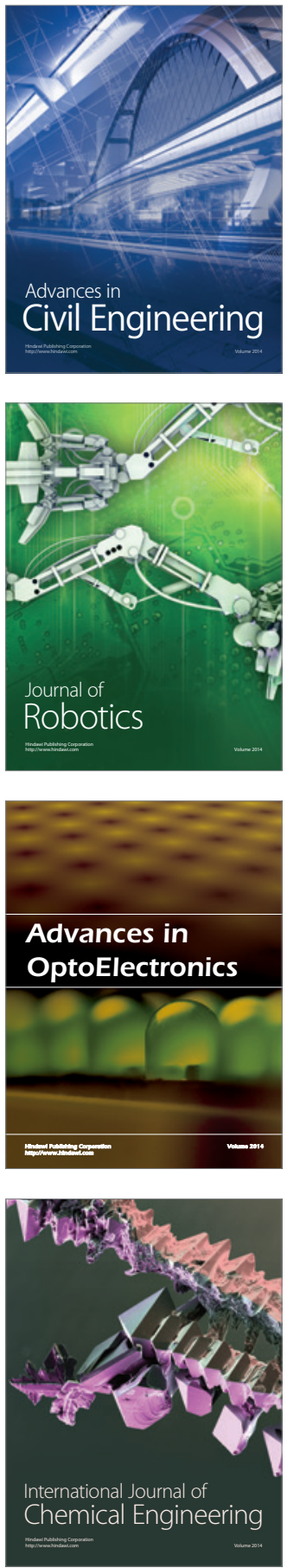

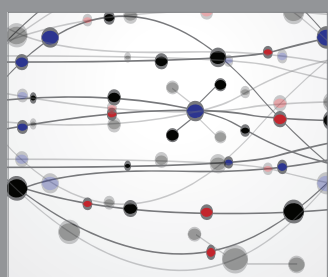

The Scientific World Journal

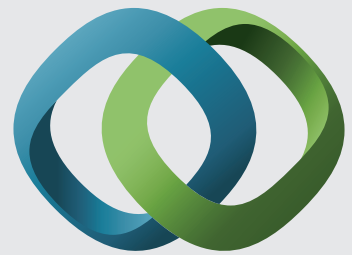

\section{Hindawi}

Submit your manuscripts at

http://www.hindawi.com
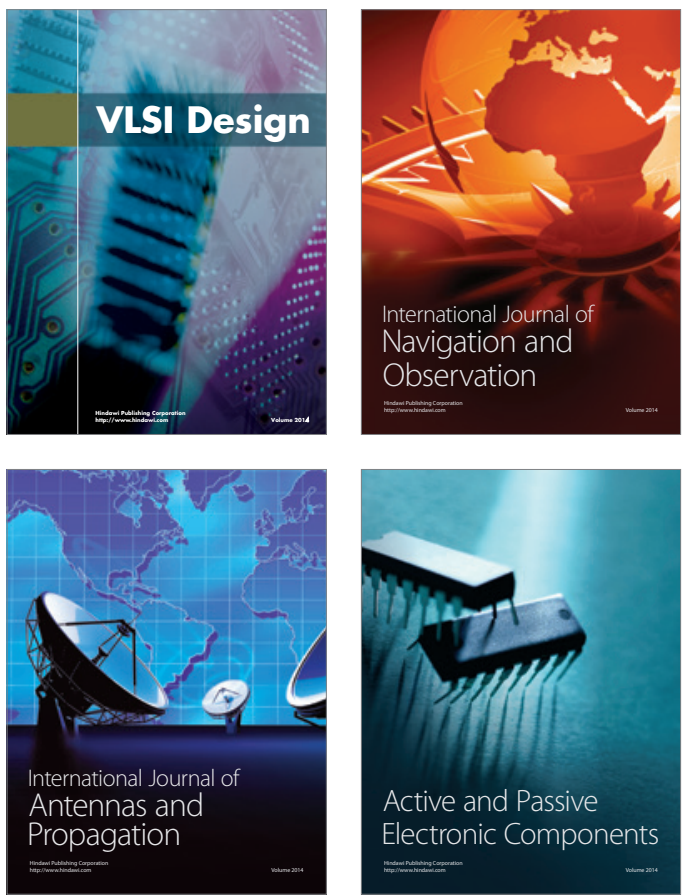
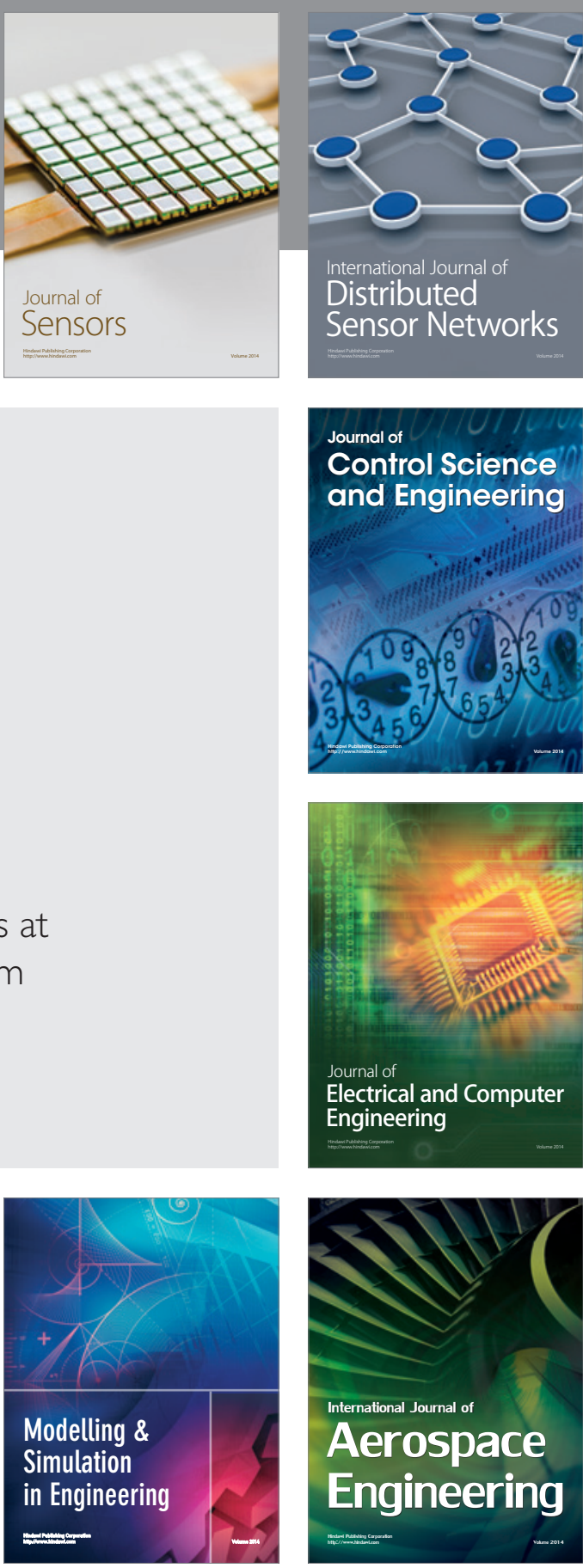

International Journal of

Distributed

Sensor Networks

Journal of

Control Science

and Engineering
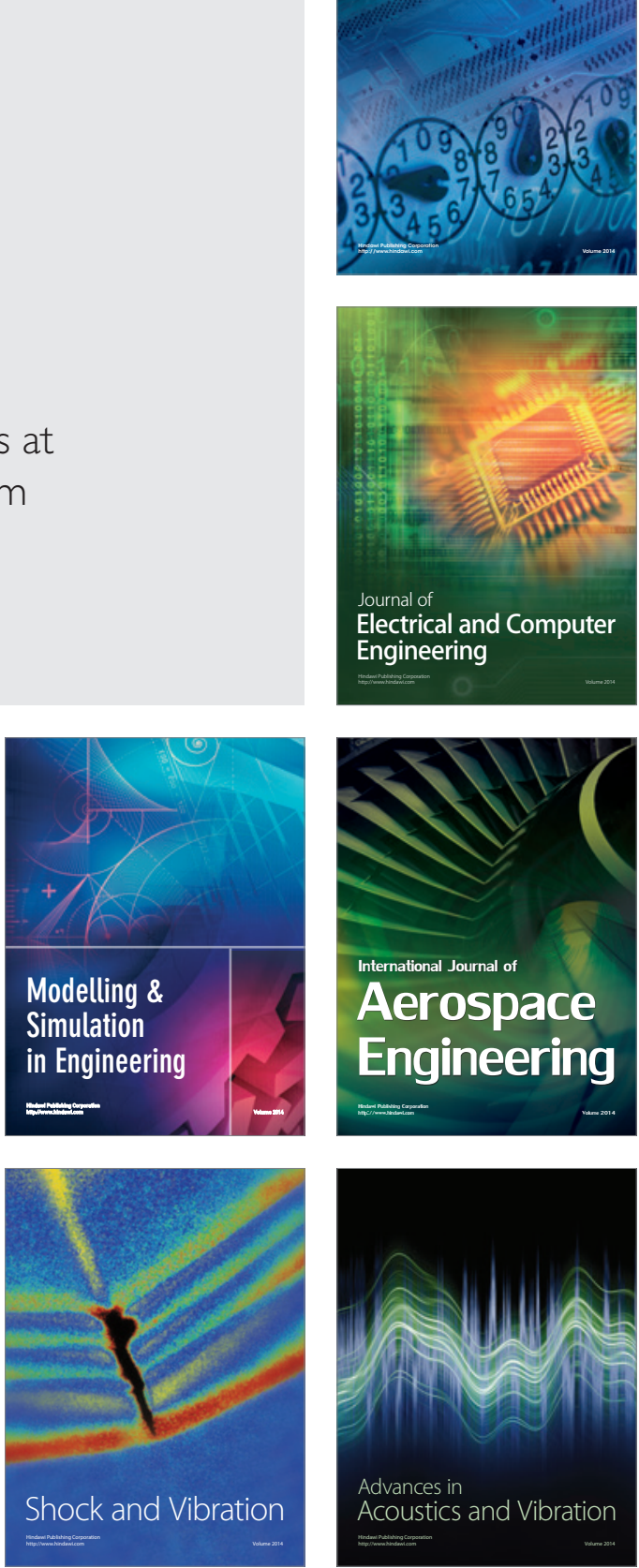